\title{
TOPOLOGICAL RIGIDITY FOR HYPERBOLIC MANIFOLDS
}

\author{
F. T. FARRELL AND L. E. JONES
}

\begin{abstract}
Let $M$ be a complete Riemannian manifold having constant sectional curvature -1 and finite volume. Let $\bar{M}$ denote its Gromov-Margulis manifold compactification and assume that the dimension of $M$ is greater than 5. (If $M$ is compact, then $\bar{M}=M$ and $\partial \bar{M}$ is empty.) We announce (among other results) that any homotopy equivalence $h:(N, \partial N) \rightarrow(\bar{M}, \partial \bar{M})$, where $N$ is a compact manifold, is homotopic to a homeomorphism. This is a topological analogue of Mostow's rigidity theorem [18]. Moreover, for each integer $j$, the surgery group $L_{j}\left(\pi_{1} M\right)$ is isomorphic to the set of homotopy classes of maps $\left[I^{k} \times \bar{M}\right.$ rel $\partial, G /$ Top $]$ where $k$ is any positive integer such that $k+\operatorname{dim} M \equiv j \bmod 4$. Here $I^{k}$ denotes the $k$-fold product $I \times I \times \cdots \times I$ where $I$ is the closed interval $[0,1]$.
\end{abstract}

Let $M$ denote a complete Riemannian manifold having constant sectional curvature -1 and finite volume. Thus $M$ is a real hyperbolic manifold of finite volume. Gromov [13] and Margulis have constructed a smooth manifold compactification of $M$ which is denoted by $\bar{M}$. Let $I^{k}$ denote the $k$-fold product $I \times I \times \cdots \times I$, where $I$ is the closed interval $[0,1]$; in particular, $I^{0}$ is a single point. Let $N$ be a compact manifold such that its boundary $\partial N$ decomposes as $\partial N=\partial_{1} N \cup \partial_{2} N$ where $\partial_{1} N, \partial_{2} N$ are compact codimension zero submanifolds of $\partial N$ with $\partial\left(\partial_{1} N\right)=\partial\left(\partial_{2} N\right)=\partial_{1} N \cap \partial_{2} N$. Set $\Lambda N=$ $\partial\left(\partial_{1} N\right)$.

THEOREM 1. Let $h:\left(N, \partial_{1} N, \partial_{2} N, \Lambda N\right) \rightarrow\left(I^{k} \times \bar{M}, \partial I^{k} \times \bar{M}, I^{k} \times \partial \bar{M}\right.$, $\left.\partial I^{k} \times \partial \bar{M}\right)$ be a homotopy equivalence of 4-tuples such that $h: \partial_{1} N \rightarrow\left(\partial I^{k}\right) \times$ $\bar{M}$ is a homeomorphism. If $k+\operatorname{dim}(M)>5$, then there is a homotopy

$h_{t}:\left(N, \partial_{1} N, \partial_{2} N, \Lambda N\right) \rightarrow\left(I^{k} \times \bar{M}, \partial I^{k} \times \bar{M}, I^{k} \times \partial \bar{M}, \partial I^{k} \times \partial \bar{M}\right), \quad t \in[0,1]$,

with $h_{0}=h, h_{1}$ a homeomorphism and the restriction of $h_{t}$ to $\partial_{1} N$ the constant homotopy. Moreover, if the restriction of $h$ to $\partial_{2} N$ is also a homeomorphism, then we need only assume that $k+\operatorname{dim}(M)>4$ and $h_{t}$ can be constructed so that it is constant on all of $\partial N$.

COROLLARY 1. Let $h:(N, \partial N) \rightarrow(\bar{M}, \partial \bar{M})$ be a homotopy equivalence of pairs where $N$ is a compact manifold. If $\operatorname{dim}(M)>5$, then there is a homotopy

$$
h_{t}:(N, \partial N) \rightarrow(\bar{M}, \partial \bar{M}), \quad t \in[0,1],
$$

Received by the editors December 13, 1987.

1980 Mathematics Subject Classification (1985 Revision). Primary 18F25, 22E40, 57D50.

Both authors were supported in part by the NSF. 
with $h_{0}=h$ and such that $h_{1}$ is a homeomorphism. Moreover, if $h: \partial N \rightarrow$ $\partial \bar{M}$ is already a homeomorphism, then we need only assume that $\operatorname{dim}(M)>4$ and $h_{t}$ can be constructed so that it is constant on $\partial N$.

Recall that each component of $\partial \bar{M}$ is homeomorphic to a flat Riemannian manifold. Hence [7] can be used to reduce the proof of Theorem 1 to the case where $h: \partial_{2} N \rightarrow I^{k} \times \partial \bar{M}$ is a homeomorphism.

Let $w_{1}: \pi_{1}(M) \rightarrow \mathbf{Z}_{2}$ denote the homomorphism determined by the first Stiefel-Whitney class of $M$, and $L_{j}\left(\pi_{1} M\right)$ denote the $j$-dimensional surgery group for the fundamental group $\pi_{1} M$ with orientation data $w_{1}$, defined by Wall in [21]. Let

$$
\Theta:\left[I^{k} \times \bar{M} \text { rel } \partial, G / \text { Top }\right] \rightarrow L_{k+m}\left(\pi_{1} M\right)
$$

be the surgery homomorphism where $m=\operatorname{dim} M$ (cf. [21 and 17]).

THEOREM 2. For each positive integer $k$, the surgery homomorphism

$$
\Theta:\left[I^{k} \times \bar{M} \text { rel } \partial, G / \text { Top }\right] \rightarrow L_{k+m}\left(\pi_{1} M\right)
$$

is an isomorphism provided $k+m>5$.

It was shown in [5 and 6] that $\Theta$ is a split monomorphism. Since $L_{j}\left(\pi_{1} M\right)$ is isomorphic to $L_{j+4}\left(\pi_{1} M\right)$ for all integers $j$, Theorem 2 yields a calculation of $L_{j}\left(\pi_{1} M\right)$ regardless of the dimension of $M$; in particular, even when $M$ is either three- or four-dimensional. Since $G /$ Top has the same rational homotopy type as does the product $\prod_{j \geq 1} K(\mathbf{Z} ; 4 j)$ of Eilenberg-Mac Lane spaces, Theorem 2 has the following consequence.

COROLLARY 2. The surgery group $L_{k+m}\left(\pi_{1} M\right) \otimes \mathbf{Q}$ is isomorphic to the direct sum of cohomology groups

$$
\bigoplus_{j=-\infty}^{\infty} H^{4 j-k}(\bar{M}, \partial \bar{M} ; \mathbf{Q})
$$

where $m=\operatorname{dim} M$ and $H^{i}(\bar{M}, \partial \bar{M} ; \mathbf{Q})$ denotes the ith cohomology group of the pair $(\bar{M}, \partial \bar{M})$ with (untwisted) rational coefficients.

The techniques used to prove Theorem 2 may also be applied to compute the surgery groups of other fundamental groups. For example, let $G$ be a finitely presented group, $\Gamma=G \times \pi_{1}(M)$ and $w_{1}: \Gamma \rightarrow \mathbf{Z}_{2}$ be a homomorphism which restricts to the identically zero homomorphism on $\pi_{1}(M)$; let $L_{j}(\Gamma)$ and $L_{j}(G)$ denote the surgery groups for the groups $\Gamma$ and $G$ with orientation data $w_{1}$ and $w_{1}$ restricted to $G$; respectively, and assume that $M$ is orientable. Let $K_{F / \text { TOP }}^{*}()$ denote the cohomology theory defined in [16].

THEOREM 3. Suppose that $\mathrm{Wh}\left(G \times C^{n}\right)=0$ holds for all nonnegative integers $n$ where $\mathrm{Wh}()$ is the Whitehead group and $C^{n}$ is the free abelian group of rank $n$. Then, for each integer $j$ the surgery group $L_{j}(\Gamma)$ is isomorphic to the sum of cohomology groups

$$
\bigoplus_{i=0}^{3} K \frac{i}{F / \mathrm{TOP}}\left(\bar{M}, \partial \bar{M} ; L_{k+i}(G)\right)
$$

where $j \equiv k+\operatorname{dim} M \bmod 4$. 
COROLlaRY 3. For all integers $j, L_{j}(\Gamma) \otimes \mathbf{Q}$ is isomorphic to the direct sum

$$
\bigoplus_{i=-\infty}^{\infty} H^{i+m}\left(\bar{M}, \partial \bar{M} ; L_{i+j}(G) \otimes \mathbf{Q}\right)
$$

where $m=\operatorname{dim} M$, provided $\mathrm{Wh}\left(G \times C^{n}\right)=0$ for all $n \geq 0$. In this formula, $H^{s}\left(\bar{M}, \partial \bar{M} ; L_{t}(G) \otimes \mathbf{Q}\right)$ denotes the s-th cohomology group of the pair $(\bar{M}, \partial \bar{M})$ with (untwisted) coefficients in $L_{t}(G) \otimes \mathbf{Q}$.

Note that Theorem 2 and Corollary 2 are the special cases of Theorem 3 and Corollary 3 where $G=1$.

Next we consider the spaces $\operatorname{Diff}(\bar{M})$ and $H(\bar{M})$ of self-diffeomorphisms and self-homeomorphisms of the smooth manifold $\bar{M}$. The following theorem is a consequence of Theorem 2 above, Theorem 4.5 in [4] (which depends on Waldhausen's foundational work in [20]), the stability results in $[\mathbf{2}, \mathbf{1 5}]$, and of the calculations of the stable pseudo-isotopy spaces given in [9].

THEOREM 4. Suppose $M$ is orientable and compact (i.e., $\partial \bar{M}$ is empty). Then, for all integers $i$ such that $1 \leq i<(m-10) / 7$, where $m=\operatorname{dim} M$, the following is true.

$$
\pi_{i} \operatorname{Diff}(M) \otimes \mathbf{Q}= \begin{cases}0 & \text { if } m \text { is even } \\ \bigoplus_{j=1}^{\infty} H_{i+1-4 j}(M, \mathbf{Q}) & \text { if } m \text { is odd. }\end{cases}
$$

More precise statements can be made about the homotopy groups of $\operatorname{Diff}(\bar{M})$ and $H(\bar{M})$; i.e., statements about $\pi_{i}$ ( ) instead of only about $\pi_{i}() \otimes \mathbf{Q}$ as in Theorem 4. This is done in the next theorem for $H(\bar{M})$ and uses [14]. Similar computations for the homotopy groups of $\operatorname{Diff}(\bar{M})$ can be found in [12].

Let $g_{1}, g_{2}, g_{3}, \ldots$ denote the sequence of all closed geodesics in $M$. (Two geodesic curves are considered equal if they have the same image in $M$.) To each closed geodesic $g_{i}$ associate a manifold $S_{i}$ as follows: $S_{i}=I^{m-1} \times S^{1}$ if $w_{1}\left(g_{i}\right)=0$, where $m=\operatorname{dim}(M)$ and $S^{1}$ is the circle; $S_{i}$ is the total space of the unorientable $I^{m-1}$ bundle over $S^{1}$ if $w_{1}\left(g_{i}\right) \neq 0$. Let $B_{1}, B_{2}, \ldots, B_{n}$ denote the connected components of $\partial \bar{M}$. For any manifold $N$, let $H(N, \partial N)$ denote the self-homeomorphisms of $N$ which are the identity on $\partial N$, and let $\hat{H}(\bar{M})$ denote the space of all $h \in H(\bar{M})$ which are homotopic (via a homotopy $H:(\bar{M}, \partial \bar{M}) \times I \rightarrow(\bar{M}, \partial \bar{M}))$ to the identity self-homeomorphism of $\bar{M}$. Define $\bigoplus_{i} H\left(S_{i}, \partial S_{i}\right)$ to be the direct limit as $j \rightarrow \infty$ of the finite cartesian products

$$
H\left(S_{1}, \partial S_{1}\right) \times H\left(S_{2}, \partial S_{2}\right) \times \cdots \times H\left(S_{j}, \partial S_{j}\right) .
$$

Define the outer automorphisms group of the pair $\left(\pi_{1} \bar{M}, \pi_{1} \partial \bar{M}\right)$ as follows. Choose base points $b_{1}, b_{2}, \ldots, b_{n}$ for each of the boundary components $B_{1}, \ldots, B_{n}$ of $\partial \bar{M}$ and a base point $b$ for $M$. By choosing paths which connect the $b_{i}$ to $b$, we establish an inclusion of $\pi_{1}\left(B_{i}, b_{i}\right)$ into $\pi_{1}(\bar{M}, b)$ for each $i=1,2, \ldots, n$. The group of outer automorphisms of the pair $\left(\pi_{1} \bar{M}, \pi_{1} \partial \bar{M}\right)$ is the quotient group Aut/In Aut. Here Aut consists of all automorphisms $f$ of $\pi_{1}(\bar{M}, b)$ such that (for each $\left.i=1,2, \ldots, n\right)$ there is an isomorphism

$$
f_{i}: \pi_{1}\left(B_{i}, b_{i}\right) \rightarrow \pi_{1}\left(B_{i^{\prime}}, b_{i^{\prime}}\right),
$$


where $i \rightarrow i^{\prime}$ is a permutation of the boundary components, and an element $g_{i}$ in $\pi_{1}(\bar{M}, b)$ satisfying $f(x)=g_{i} f_{i}(x) g_{i}^{-1}$ for all $x$ in $\pi_{1}\left(B_{i}, b_{i}\right)$. The normal subgroup InAut of Aut is the ordinary inner automorphism group of $\pi_{1}(\bar{M}, b)$.

THEOREM 5. (a) There is a bijection between the set of homotopy classes of self-homeomorphisms of $\bar{M}$ and the outer automorphism group of $\left(\pi_{1} \bar{M}, \pi_{1} \partial \bar{M}\right)$. (Here homotopy classes refer to homotopies of maps of the pair $(\bar{M}, \partial \bar{M})$.)

(b) There is a continuous map

$f: H\left(B_{1} \times I, B_{1} \times 0\right) \times \cdots \times H\left(B_{n} \times I, B_{n} \times 0\right) \times\left(\bigoplus_{i} H\left(S_{i}, \partial S_{i}\right)\right) \rightarrow \hat{H}(\bar{M})$

which is induced by the inclusions $g_{i} \subset M$ and $B_{j} \subset \bar{M}$, where $i>0$ and $j=1,2, \ldots, n$. The map $f$ induces an isomorphism on the homotopy groups $\pi_{i}$, provided $i<\phi(\operatorname{dim} M)$, where $\phi: \mathbf{R} \rightarrow \mathbf{R}$ is a function (independent of M) such that $\lim _{m \rightarrow+\infty} \phi(m)=+\infty$.

COROLLARY 5. The vanishing statement

$$
\pi_{i} H(\bar{M}) \otimes \mathbf{Q}=0
$$

is true provided $1 \leq i<\phi(m)$, where $m$ denotes the dimension of $M$.

ADDED IN PROOF. Because of a result of Goodwillie, the function $\phi(m)$ satisfies the inequality $\phi(m) \geq(m-10) / 7$; this lower bound and the "stable range" in Theorem 4 can be greatly improved using Igusa's recent work [22].

REMARK. There are analogues to Theorems $1,2,3$ and $5(\mathrm{~b})$ when $M$ is not assumed to have finite volume. These analogues require the following changes. All homotopy equivalences of Theorem 1 must now have compact support; i.e., must be an embedding away from a large enough compact set. The set $\left[I^{k} \times \bar{M}\right.$ rel $\partial, G /$ Top] of Theorem 2 must be replaced by the set of compactly supported homotopy equivalence classes of compactly supported maps. The cohomology groups of Theorem 3 must be replaced by cohomology with compact support. The spaces $H($ ) and $\hat{H}()$ of Theorem $5(\mathrm{~b})$ must be replaced by the spaces of compactly supported self-homeomorphisms and the homotopy $H$ must have compact support.

Theorems 1-5 are proved in [12]. In sequels to this paper we will pursue analogoues of Theorem 1-5 for wider classes of manifolds, including nonpositively curved locally symmetric spaces of higher rank.

We give now an indication of the proof for the special case of Theorem 1 given in Corollary 1. Our proof follows the same general outline as the arguments given in papers [8 and 9], in which the lower algebraic $K$-groups of the fundamental group of any real hyperbolic manifold were computed together with a determination of the weak homotopy type of its stable pseudoisotopy space. There are two major ingredients:

(1) a foliated control theorem for structure spaces,

(2) the dynamics of the geodesic flow (cf. [1]) together with other geometric ideas. 
The foliated control theorem for structure spaces, used in the proof of Theorem 1, is proved in [11]. It extends the unfoliated control results of [3 and 19]. Unlike the arguments in papers $[8,9$ and 10] where we needed only foliated control theorems for foliations having one-dimensional leaves, the proof of Theorem 1 requires a structure space foliated control theorem for foliations having higher-dimensional leaves.

So as to abbreviate the many complications which arise in the proof, we assume that $M$ is an odd-dimensional manifold The geometry of $M$ enters into the proof of Theorem 1 in the following way. It enables us to construct a compact stratified space $F$ with two strata $B$ and $T$ where $B$ is the bottom stratum and $T$ is the top stratum. The fundamental group $\Gamma$ of $M$ acts on $F$ preserving these strata. Let $F(M)$ denote the orbit space of $\tilde{M} \times F$ under the diagonal action of $\Gamma$ where $\tilde{M}$ is the universal cover of $M$; i.e.,

$$
F(M)=\tilde{M} \times_{\Gamma} F .
$$

Note $F(M)$ is the total space of a fiber bundle over $M$ with fiber $F$. Thus it is a stratified space having two strata $\mathbf{B}$ and $\mathbf{T}$ where

$$
\mathbf{B}=\tilde{M} \times_{\Gamma} B \quad \text { and } \quad \mathbf{T}=\tilde{M} \times_{\Gamma} T .
$$

Furthermore $F(M)$ is equipped with a foliation $\mathscr{F}$ which restricts to smooth foliations $\mathscr{F}_{B}$ and $\mathscr{F}_{T}$ for the strata $\mathbf{B}$ and $\mathbf{T}$, respectively. The geometry of $M$ gives rise to additional structures, called markings, on the leaves of $\mathscr{F}_{B}$ and $\mathscr{F}_{T}$. Each leaf of $\mathscr{F}_{T}$ is marked with a geodesic while each leaf of $\mathscr{F}_{B}$ is marked with an asymptotic vector field. These markings vary smoothly from leaf to leaf within each stratum.

Consider a homotopy equivalence $h: N \rightarrow \bar{M}$ from a compact manifold $N$ which restricts to a homeomorphism near $h: \partial N \rightarrow \partial \bar{M}$. (See the comment following the statement of Corollary 1.) Thus $h: N-\partial N \rightarrow M$ is a homotopy equivalence with compact support. Let $F^{*}$ denote the total space of the pullback of the bundle $F(M) \rightarrow M$ along $h$ restricted to $N-\partial N$, and let $h^{*}: F^{*} \rightarrow F(M)$ denote the natural lifting of $h: N-\partial N \rightarrow M$ to $F^{*}$. We claim there is a compactly supported homotopy $h_{t}^{*}, t \in[0,1]$, with $h_{0}^{*}=h^{*}$ and such that $h_{1}^{*}$ is a homeomorphism over $\mathbf{B}$ and over the complement of a tubular neighborhood for the stratum $\mathbf{B}$ in $F(M)$, and such that $h_{1}^{*}$ is "blocked up" over this tubular neighborhood. To get such a homotopy, we first "flow" $h^{*}$ over the bottom stratum in the direction of the asymptotic vector fields of the leaves of $\mathscr{F}_{B}$, thereby gaining one-dimensional foliated control for $h^{*}$ over the bottom stratum B. Now apply the structure space foliated control theorem from [10 and 11] (for the one-dimensional foliation of $\mathbf{B}$ consisting of the flow lines) to construct the homotopy $h_{t}^{*}$ over $\mathbf{B}$. To get $h_{t}^{*}$ over the top stratum (away from a tabular neighborhood of $\mathbf{B}$ ), we use the geodesic markings on the leaves of $\mathscr{F}_{T}$ together with the structure space foliated control theorem for higher dimensional leaves of special geometric type, proved in [11]. Finally, $h_{t}^{*}$ is extended over the tubular neighborhood of $\mathbf{B}$ by the fibered version of the foliated control theorem for foliations with one-dimensional leaves, also proved in [11]. 
Surgery procedures now allow us to "desuspend" the homotopy $h_{t}^{*}$ of the preceding paragraph to get a homotopy $h_{t}: N \rightarrow \bar{M}, t \in[0,1]$, from $h=h_{0}$ to a homeomorphism $h_{1}$ and such that $h_{t}$ restricted to $\partial N$ is the constant homotopy. It is at this step that we use the extra assumption: $M$ has odd dimension, which implies $F$ has index one.

\section{REFERENCES}

1. D. V. Anosov, Geodesic flows on closed Riemannian manifolds with negative curvature, Proc. Steklov Inst. Math. 90 (1969).

2. D. Burghelea and R. Lashof, Stability of concordances and the suspension homomorphism, Ann. of Math. (2) 105 (1977), 449-472.

3. T. A. Chapman and S. Ferry, Approximating homotopy equivalences by homeomorphisms, Amer. J. Math. 101 (1979), 567-582.

4. F. T. Farrell and W. -C. Hsiang, On the rational homotopy groups of the diffeomorphism groups of discs, spheres, and aspherical manifolds, Proc. Sympos. Pure Math. vol. 32, Amer. Math. Soc., Providence, R. I., 1978, pp. 325-337.

5. _ On Novikov's conjecture for non-positively curved manifolds. I, Ann. of Math. (2) 113 (1981), 199-209.

6. __ The stable topological-hyperbolic space form problem for finite volume manifolds, Invent. Math. 69 (1982), 155-170.

7. - Topological characterization of flat and almost flat Riemannian manifolds $M^{n}$ ( $n \neq$ 3,4), Amer. J. Math. 105 (1983), 641-672.

8. F. T. Farrell and L. E. Jones, K-theory and dynamics. I, Ann. of Math. (2) 124 (1986), 531-569.

9. __ K-theory and dynamics. II, Ann. of Math. (2) 126 (1987), 451-493.

10. The surgery L-groups of poly-(finite or cyclic) groups, Invent. Math. (1988).

11. _ Foliated control theory. I and II, submitted for publication.

12. _ Topological analogue of Mostow's rigidity theorem, in preparation.

13. M. Gromov, Manifolds of negative curvature, J. Differential Geom. 13 (1978), 223-230.

14. A. E. Hatcher, Concordance spaces, higher simple homotopy theory, and applications, Proc. Sympos. Pure Math., vol. 32, Amer. Math. Soc., Providence, R. I., 1978, pp. 3-21.

15. K. Igusa, Stability of pseudoisotopies, Seminar Notes 1, Aarhus Univ., Matematisk Institut, Aarhus, Denmark, 1982, pp. 27-37.

16. L. E. Jones, The nonsimply connected characteristic variety theorem, Proc. Sympos. Pure Math., vol 32, Amer. Math. Soc., Providence, R. I., 1978, pp. 131-140.

17. R. C. Kirby and L. C. Siebenmann, Foundational essays on topological manifolds, smoothings, and triangulations, Ann. of Math. Studies, Princeton Univ. Press, Princeton, N. J., 1977.

18. G. D. Mostow, Quasi-conformal mappings in n-space and the rigidity of hyperbolic space forms, Publ. Inst. Hautes Études Sci. 34 (1967), 53-104.

19. F. Quinn, Ends of maps. I, Ann. of Math. (2) 110 (1979), 275-331.

20. F. Waldhausen, Algebraic K-theory of topological spaces. I, Proc. Sympos. Pure Math., vol 32, Amer. Math. Soc., Providence, R. I., 1978, pp. 35-60.

21. C. T. C. Wall, Surgery on compact manifolds, Academic Press, New York, 1970.

22. K. Igusa, The stability theorem for pseudoisotopies, preprint.

Department of Mathematics, Columbia University, NeW York, NeW YORK 10027

Department of Mathematics, State University of NeW York, Stony BROOK, NEW YORK 11794 